\title{
Toward more accurate documentation in neurosurgical care
}

\author{
Rohaid Ali, MD,, Sohail Syed, MD, ${ }^{1}$ Rahul A. Sastry, MD, ${ }^{1}$ Hael Abdulrazeq, MD, ${ }^{1}$ \\ Belinda Shao, MD, MPH, ${ }^{1}$ G. Dean Roye, MD, ${ }^{2}$ Curtis E. Doberstein, MD, ${ }^{1}$ \\ Adetokunbo Oyelese, MD, PhD, ${ }^{1}$ Tianyi Niu, MD, ${ }^{1}$ Ziya L. Gokaslan, MD, ${ }^{1}$ and \\ Albert Telfeian, MD, PhD'
}

\begin{abstract}
${ }^{1}$ Department of Neurosurgery, Warren Alpert Medical School of Brown University; and ${ }^{2}$ Department of Surgery, Warren Alpert Medical School of Brown University, Providence, Rhode Island
\end{abstract}

OBJECTIVE Accurate clinical documentation is foundational to any quality improvement endeavor as it is ultimately the medical record that is measured in assessing change. Literature on high-yield interventions to improve the accuracy and completeness of clinical documentation by neurosurgical providers is limited. Therefore, the authors sought to share a single-institution experience of a two-part intervention to enhance clinical documentation by a neurosurgery inpatient service.

METHODS At an urban, level I trauma, academic teaching hospital, a two-part intervention was implemented to enhance the accuracy of clinical documentation of neurosurgery inpatients by residents and advanced practice providers (APPs). Residents and APPs were instructed on the most common neurosurgical complications or comorbidities (CCs) and major complications or comorbidities (MCCs), as defined by Medicare. Additionally, a "system-based" progress note template was changed to a "problem-based" progress note template. Prepost analysis was performed to compare the CC/MCC capture rates for the 12 months prior to the intervention with those for the 3 months after the intervention.

RESULTS The CC/MCC capture rate for the neurosurgery service line rose from $62 \%$ in the 12 months preintervention to $74 \%$ in the 3 months after intervention, representing a significant change $(p=0.00002)$.

CONCLUSIONS Existing clinical documentation habits by neurosurgical residents and APPs may fail to capture the extent of neurosurgical inpatients with CC/MCCs. An intervention that focuses on the most common CC/MCCs and utilizes a problem-based progress note template may lead to more accurate appraisals of neurosurgical patient acuity.

https://thejns.org/doi/abs/10.3171/2021.8.FOCUS21387

KEYWORDS quality improvement; complication; comorbidity; CC/MCC; documentation

\section{$\mathrm{T}$} HE modern-day neurosurgeon is increasingly tasked with enacting value-based care, which is the principle of delivering healthcare with both quality and cost in mind. ${ }^{1}$ Central to this endeavor is accurate clinical documentation, a crucial element to informing any meaningful quality improvement initiative, and it is also important for justification of costs in what is often a resourceintensive specialty.

As a brief background, in 1983, the Centers for Medicare and Medicaid Services created the inpatient Prospective Payment System (PPS), a framework for documenting patient diagnoses and comorbidities. ${ }^{2}$ The PPS consists of Diagnosis Related Groups (DRGs) - groupings of diseases with similar treatment pathways-and since its in- ception, it has undergone more than 30 revisions. In 2007, a significant change occurred to the PPS in the form of the Medicare Severity DRGs (MS-DRGs). ${ }^{3}$ This revision introduced a two- and three-tiered classification system for diagnoses within a DRG based on the level of severity; these are called complications or comorbidities (CCs) and major complications or comorbidities (MCCs). Within a DRG the lowest acuity diagnosis is classified as non-CC, the middle acuity diagnosis as $\mathrm{CC}$, and the highest acuity diagnosis as MCC.

In an era that focuses increasingly on value-based care, attention to quality and cost is a must for the neurosurgical community. ${ }^{1}$ Neurosurgery is especially prone to adverse events compared with other surgical subspecialities,

ABBREVIATIONS APP = advanced practice provider; $C C=$ complication or comorbidity; DRG = Diagnosis Related Group; $M C C=$ major complication or comorbidity; $M S$ DRG = Medicare Severity DRG; PPS = Prospective Payment System; UHC = University HealthSystem Consortium .

SUBMITTED June 30, 2021. ACCEPTED August 24, 2021.

INCLUDE WHEN CITING DOI: 10.3171/2021.8.FOCUS21387. 
and certain hospitals such as level I trauma centers and academic teaching hospitals may receive higher acuity patients compared with other types of institutions. ${ }^{4-9}$ Accurate clinical documentation is tantamount to capturing the value of healthcare delivery in this context. Without it, it would be impossible to perform reliable, risk-adjusted multiinstitutional comparisons and single-site quality improvement endeavors..$^{10}$ These comparisons and endeavors can also affect the public image of a hospital or clinician, such as through national hospital rankings. Moreover, appropriate documentation of $\mathrm{CC} / \mathrm{MCCs}$ affects cost-impacting healthcare metrics such as severity of illness, risk of mortality, case mix index, and projected length of stay. These, in turn, have a direct effect on facility reimbursement, and failure to document a CC or an MCC can lead to loss of reimbursement for a facility. ${ }^{11}$ Despite the influence of accurate clinical documentation on both healthcare quality and cost, limited attention has been paid in the neurosurgical literature on low-cost, high-yield interventions to enhance clinical documentation. We seek to share our experience of implementing a two-part intervention to enhance clinical documentation by a neurosurgery inpatient service at an urban, level I trauma, academic teaching hospital.

\section{Methods \\ Setting}

This single-center initiative was conducted on the neurosurgery inpatient service of Rhode Island Hospital, a 700-bed tertiary care, academic teaching hospital that also serves as the sole level I trauma center in the southern New England region. The inpatient service includes both elective admissions for scheduled surgery and emergency department admissions and direct admissions for nonambulatory neurosurgical acute care. Given this widely varied patient population and high acuity of patients who present at such a center, we suspected the opportunity to capture $\mathrm{CC} / \mathrm{MCCs}$ would be vast.

\section{Interventions}

Two interventions were introduced to enhance the clinical documentation habits of the neurosurgical residents and advanced practice providers (APPs). First, the residents and APPs were taught, in December 2020, the 4 most common CC/MCCs for neurosurgical patients affected by cranial issues (e.g., brain tumors and traumatic brain injury); these were brain compression, cerebral edema, brain herniation, and encephalopathy. This instruction was done by a neurosurgical resident with the assistance and guidance of a clinical documentation specialist. Second, the neurosurgical inpatient service at this hospital historically used a system-based progress note template to capture clinical data and delineate daily plans. However, the system-based template often lacked documentation of the specific problems that were being addressed and treated. Specifically, these notes focused on plans addressing the patient's neurological, pulmonary, and cardiac systems, among others, but failed to include an assessment of the underlying conditions with which a patient presented. This often led to missing essential CC/MCCs and failure to capture the acuity of the patients on the neurosurgery service. Therefore, the system-based progress note template was converted to a problem-based progress note template. The focus of the new notes was to include active problems and diagnoses, in addition to problems that are present on admission (e.g., congestive heart failure and diabetes mellitus). This was done in an effort to make the problems that were being addressed for a patient during an admission more explicit and to record what the specific treatment plans were for each problem.

\section{Statistical Analysis}

A 12-month period prior to the intervention was compared with a 3-month period postintervention. The principal data point examined was the $\mathrm{CC} / \mathrm{MCC}$ capture rate, which represents the percentage of patients with a secondary diagnosis of a CC and/or an MCC during a hospitalization. These data were provided by the University HealthSystem Consortium (UHC), a member-driven consortium representing half of healthcare facilities in the United States. ${ }^{12}$ The CC/MCC capture rate can be obtained by the service line for each hospital. We chose to obtain the $\mathrm{CC} / \mathrm{MCC}$ capture rate for the neurosurgery service line and also the spinal surgery service line. Of note, the spinal surgery service line at our hospital is a shared service line between the orthopedics and neurosurgery departments.

Data for the neurosurgery and spinal surgery service lines for the 12 months prior to intervention and 3 months postintervention were obtained. Outcomes included the $\mathrm{CC} / \mathrm{MCC}$ capture rate as well as the national percentiles for these captures in the UHC database. The t-test was used for statistical comparison, with a significance level of $\mathrm{p} \leq 0.05$.

\section{Results}

During the 12 months prior to intervention, the average CC/MCC capture rate for the neurosurgery service line was $62 \%$ (Fig. 1). During the 3 months after intervention, the average $\mathrm{CC} / \mathrm{MCC}$ capture rate was $74 \%$, representing a significant increase $(\mathrm{p}=0.00002)$. For the spinal surgery service line 12 months prior to intervention, the average $\mathrm{CC} / \mathrm{MCC}$ capture rate was $40 \%$; after intervention, the CC/MCC capture rate rose to $48 \%$, also representing a significant increase $(\mathrm{p}=0.001515)$. Additionally, prior to the intervention, the neurosurgery service line was performing below the 25th percentile compared with the national CC/MCC capture rates, according to the UHC. After the intervention, the neurosurgery service line rose to between the 50th and 75th percentiles nationally for the $\mathrm{CC} / \mathrm{MCC}$ capture rate.

\section{Discussion}

Herein, we demonstrate how a low-cost, high-yield intervention can improve the $\mathrm{CC} / \mathrm{MCC}$ capture rate for the neurosurgery and spinal surgery service lines at an urban, level I trauma, academic teaching hospital. Educating residents and APPs on the 4 most common CC/MCCs for the neurosurgery service line and switching from a systembased to a problem-based progress note template led to 

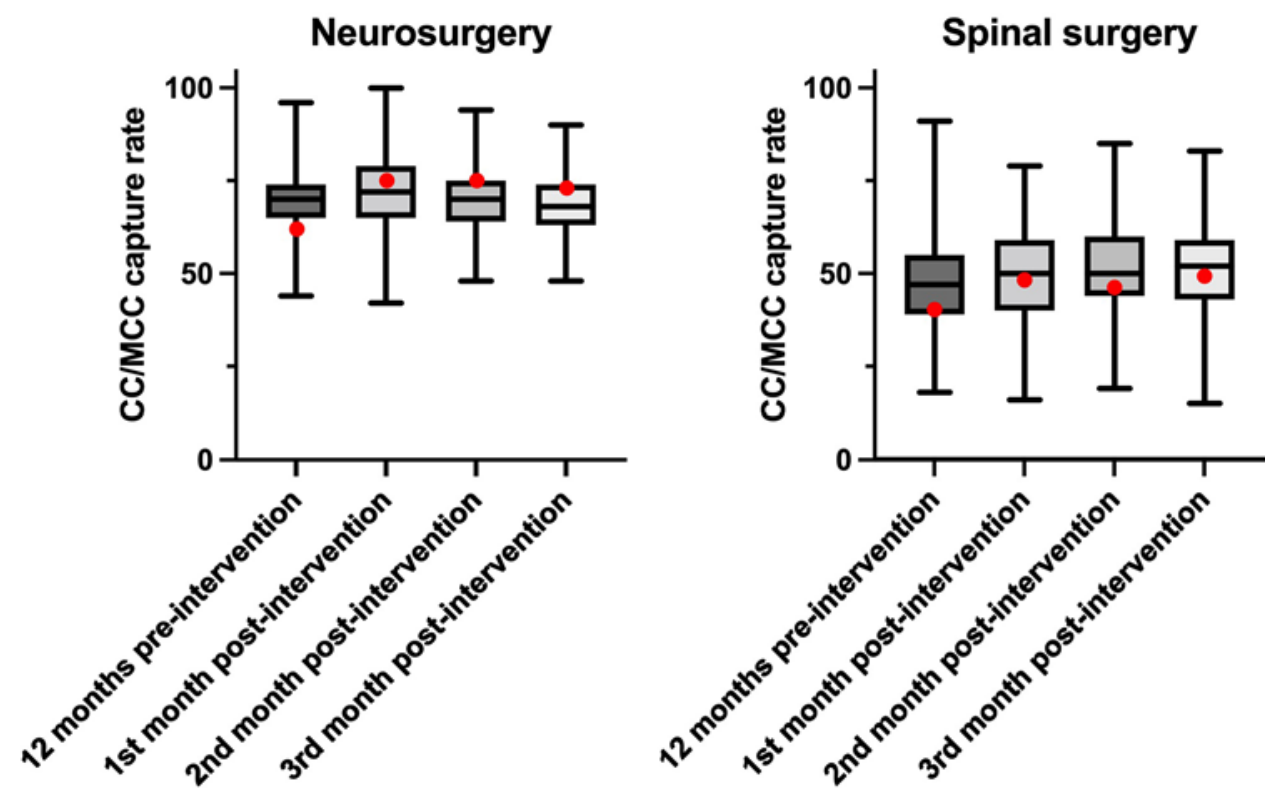

FIG. 1. Boxplots showing the national values for CC/MCC capture rates including the minimum, 25th percentile, median, 75th percentile, and maximum. The red dots signify the institutional CC/MCC capture rate for the neurosurgery and spinal surgery service lines 12 months preintervention and each month for 3 months postintervention. Left: For the neurosurgery service line, the preintervention $\mathrm{CC} / \mathrm{MCC}$ capture rate average was $62 \%$, below the national 25 th percentile of $65 \%$; following intervention, the average $\mathrm{CC} / \mathrm{MCC}$ capture rate for neurosurgery rose to $74 \%$, close to the 75 th percentile nationally of $76 \%$. Right: For the spinal surgery service line, the preintervention CC/MCC capture rate was 40\%; postintervention, the average CC/MCC capture rate for spinal surgery rose to $48 \%$. Of note, the spinal surgery service line comprises orthopedics and neurosurgery and the specific interventions were not targeted to the spine surgery service line and were not adopted in the orthopedics department (see Discussion for further information).

significant increases in the $\mathrm{CC} / \mathrm{MCC}$ capture rates for both the neurosurgery and spinal surgery service lines. This work demonstrates how relatively minor adjustments to clinical documentation habits can lead to big changes in accurately capturing neurosurgical patient acuity and, thereby, better representing the value of healthcare delivered.

There have been other published reports of clinical documentation enhancement initiatives at academic programs. ${ }^{13-16}$ One academic general surgery department implemented an ICD-10 training initiative that decreased delinquent documentation by $85 \%$ and in turn resulted in more than \$4 million of increased estimated reimbursement, as well as significant increases in metrics such as severity of illness, case mix index, risk of mortality, and compliance with national surgical quality improvement scores. ${ }^{11}$ A 2013 study found that an academic program including lectures and reference cards for residents in internal medicine did have a demonstrative effect on the $\mathrm{CC} / \mathrm{MCC}$ capture rate, mortality index, and case mix index over a 2-year period. ${ }^{15}$ Similarly, in 2016, an academic medical center's department of otolaryngology shared results of its clinical documentation enhancement initiative, which also consisted of lectures and flash cards for residents and attendings; this work yielded significant increases in the severity of illness and risk of mortality scores as well as the CC/MCC capture rate. ${ }^{16}$ Another academic medical center's neurosurgery department found success when it implemented a clinical documentation enhancement program that consisted of seven parts, includ- ing monthly individualized emails, grand rounds sessions, individualized feedback sessions, and more. ${ }^{13}$

Our intervention's switch from a system-based to a problem-based progress note template is based on literature that found that problem-based charting leads to more complete capturing of patient diagnoses. A 2017 study found that implementation of a problem-based charting system increased the total number of problems per patient by more than two, and it also found that accuracy of documenting such problems as sepsis and acute renal failure improved. ${ }^{17}$ Furthermore, as part of a quality improvement initiative at an academic medical center's gynecology and obstetrics department, researchers found that incorporating a problem-based charting system (among other interventions) improved accuracy in severity of illness scores. ${ }^{14}$ Our findings further bolster the notion that problem-based charting, as opposed to system-based, improves accuracy and completeness of clinical documentation.

The decision to list just $4 \mathrm{CC} / \mathrm{MCC}$ as the focus for neurosurgical patients was due to several reasons, most notably the time constraints facing providers. Indeed, a prior clinical documentation enhancement effort in 2011 attempted to use an exhaustive, multisystem checklist for clinical comorbidities; unfortunately, this effort was met with no success in improving severity of illness scores for patients after intervention..$^{8}$ The authors acknowledged that it was difficult having physicians adapt to this quality improvement initiative. We suspect this may have been due, in part, to the exhaustive nature of the checklist, so we sought to avoid this problem in our clinical documen- 
tation intervention by introducing a more digestible collection of problems to ask providers to more consistently identify and document. Furthermore, the new progress note template included a feature by which problems that were active in the electronic medical record would autofill within the note. Thus, the provider in many instances only had to click through a historic problem list within the chart or add additional problems that may have developed postoperatively. As a result, these problems were automatically populated within the clinical documentation. This led to an increase in efficiency in documentation of problems, and capturing more of them in less time, which was essential on a historically busy service line.

Interestingly, we found that our spinal surgery service line CC/MCC capture rate had a significant increase. This occurred despite the fact that none of the $4 \mathrm{CC} / \mathrm{MCCs}$ emphasized at the start of the intervention were relevant for spine patients and also despite the fact that spine surgery at this hospital is a shared service line between neurosurgery and orthopedic surgery, a service with different clinical documentation habits and whose providers were not a part of this intervention. This may be in part due to the complexity of medical issues that are comorbid within the population of patients undergoing spinal surgery in many instances. From this we glean that the shift to a problem-based progress note template for just the neurosurgery service may have been enough to improve the CC/MCC capture rate for spinal surgery significantly; the fact that the neurosurgery service line rose more in both absolute terms and relative to national percentiles may be because in addition to switching to a problembased charting system we also educated providers on the 4 most common CC/MCCs applicable to cranial neurosurgical patients. Perhaps identifying the most common $\mathrm{CC} / \mathrm{MCC}$ for spinal surgery and educating providers on them would lead to a larger increase in the relative $\mathrm{CC} /$ MCC capture rate.

One element that separates this work from the aforementioned literature is the lower-resource nature of our clinical documentation enhancement initiative. We did not use flash cards, a lecture series, or individualized feedback sessions. Rather, just by focusing on a handful of "can't miss" CC/MCCs as well as moving from a system-based to a problem-based charting system, we saw a dramatic increase in our $\mathrm{CC} / \mathrm{MCC}$ capture rate for both neurosurgery and spinal surgery service lines. The implications of this are twofold. First, the low-cost, high-yield changes that we implemented may be the biggest drivers of change in health metrics seen in other studies that incorporated a more exhaustive quality improvement approach. Second, our approach may be more feasible for hospitals and programs with smaller clinical documentation improvement programs.

\section{Conclusions}

Existing clinical documentation patterns by neurosurgical providers may fail to fully capture patient acuity and, thereby, the value of care delivered. However, in an era where hospital systems are increasingly striving for value-based care models, the onus is on neurosurgeons to deliver. The results of this study have demonstrated a lowcost, high-yield intervention that greatly improved the $\mathrm{CC} /$ MCC capture rates for the neurosurgery and spinal surgery service lines at an urban, level I trauma, academic teaching hospital.

\section{References}

1. McLaughlin N, Upadhyaya P, Buxey F, Martin NA. Valuebased neurosurgery: measuring and reducing the cost of microvascular decompression surgery. J Neurosurg. 2014; 121(3):700-708.

2. Iglehart JK. Medicare begins prospective payment of hospitals. N Engl J Med. 1983;308(23):1428-1432.

3. Centers for Medicare and Medicaid Services (CMS), HHS. Medicare program; changes to the hospital inpatient prospective payment systems and fiscal year 2008 rates. Fed Regist. 2007;72(162):47129-48175.

4. Wong JM, Bader AM, Laws ER, Popp AJ, Gawande AA. Patterns in neurosurgical adverse events and proposed strategies for reduction. Neurosurg Focus. 2012;33(5):E1.

5. Wong JM, Panchmatia JR, Ziewacz JE, Bader AM, Dunn IF, Laws ER, Gawande AA. Patterns in neurosurgical adverse events: intracranial neoplasm surgery. Neurosurg Focus. 2012;33(5):E16.

6. Wong JM, Ziewacz JE, Ho AL, Panchmatia JR, Bader AM, Garton HJ, et al. Patterns in neurosurgical adverse events: cerebrospinal fluid shunt surgery. Neurosurg Focus. 2012; 33(5):E13.

7. Wong JM, Ziewacz JE, Ho AL, Panchmatia JR, Kim AH, Bader AM, et al. Patterns in neurosurgical adverse events: open cerebrovascular neurosurgery. Neurosurg Focus. 2012; 33(5):E15.

8. Wong JM, Ziewacz JE, Panchmatia JR, Bader AM, Pandey AS, Thompson BG, et al. Patterns in neurosurgical adverse events: endovascular neurosurgery. Neurosurg Focus. 2012; 33(5):E14.

9. Houkin K, Baba T, Minamida Y, Nonaka T, Koyanagi I, Iiboshi S. Quantitative analysis of adverse events in neurosurgery. Neurosurgery. 2009;65(3):587-594.

10. Grogan EL, Speroff T, Deppen SA, Roumie CL, Elasy TA, Dittus RS, et al. Improving documentation of patient acuity level using a progress note template. J Am Coll Surg. 2004; 199(3):468-475.

11. Reyes C, Greenbaum A, Porto C, Russell JC. Implementation of a clinical documentation improvement curriculum improves quality metrics and hospital charges in an academic surgery department. J Am Coll Surg. 2017;224(3):301-309.

12. Hammers R, Anzalone S, Sinacore J, Origitano TC. Neurosurgical mortality rates: what variables affect mortality within a single institution and within a national database? $J$ Neurosurg. 2010;112(2):257-264.

13. Elsamadicy AA, Sergesketter A, Sampson JH, Gottfried ON. Institutional review of mortality in 5434 consecutive neurosurgery patients: are we improving? Neurosurgery. 2018; 83(6):1269-1276.

14. Arquiette JM, Moss HA, Truong T, Pieper CF, Havrilesky LJ. Impact of a documentation intervention on health-assessment metrics on an inpatient gynecologic oncology service. Gynecol Oncol. 2019;153(2):385-390.

15. Spellberg B, Harrington D, Black S, Sue D, Stringer W, Witt M. Capturing the diagnosis: an internal medicine education program to improve documentation. Am J Med. 2013;126(8): 739-743.e1.

16. Momin SR, Lorenz RR, Lamarre ED. Effect of a documentation improvement program for an academic otolaryngology practice. JAMA Otolaryngol Head Neck Surg. 2016;142(6): 533-537. 
17. Li RC, Garg T, Cun T, Shieh L, Krishnan G, Fang D, Chen JH. Impact of problem-based charting on the utilization and accuracy of the electronic problem list. J Am Med Inform Assoc. 2018;25(5):548-554.

18. Spurgeon A, Hiser B, Hafley C, Litofsky NS. Does improving medical record documentation better reflect severity of illness in neurosurgical patients? Clin Neurosurg. 2011;58: 155-163.

\section{Disclosures}

The authors report no conflict of interest concerning the materials or methods used in this study or the findings specified in this paper.

\section{Author Contributions}

Conception and design: Ali, Niu, Gokaslan, Telfeian. Acquisition of data: Ali, Syed, Sastry, Abdulrazeq, Shao. Analysis and interpretation of data: Ali, Roye, Telfeian. Drafting the article: Ali. Critically revising the article: Ali, Syed, Abdulrazeq, Shao, Roye, Gokaslan, Telfeian. Reviewed submitted version of manuscript: all authors. Approved the final version of the manuscript on behalf of all authors: Ali. Statistical analysis: Ali. Administrative/ technical/material support: Roye, Doberstein, Niu, Gokaslan, Telfeian. Study supervision: Roye, Gokaslan, Telfeian.

\section{Correspondence}

Rohaid Ali: Warren Alpert Medical School of Brown University, Rhode Island Hospital, Providence, RI. rali@lifespan.org. 